\title{
Pontas tipo cone com variação da taxa de aplicação e velocidade na cultura do algodoeiro
}

\author{
Thiago Martins MACHADO ${ }^{1 *}$, Monyse Fin Barbosa ${ }^{1}$, \\ Adelina Vitória Servelhere de REZENDE ${ }^{1}$, Ronan Sauer BUENO ${ }^{1}$
}

${ }^{1}$ Instituto de Ciências Agrárias e Ambientais, Universidade Federal de Mato Grosso, Sinop, MT, Brasil.

E-mail: tmmachado@ufmt.br

Recebido em outubro/2018; Aceito em fevereiro/2019.

RESUMO: A Ramulária é uma das principais doenças fungicas do algodoeiro, trazendo consequências graves para sua produtividade e qualidade de fibras. Com o intuito de abater os gastos e melhorar os parâmetros da aplicação, o trabalho tem por objetivo avaliar diferentes tipos de pontas de jato cônico e pingente, variando a taxa de aplicação e velocidade na pulverização do algodoeiro. Foram utilizadas pontas de jato cone vazio e cheio de diferentes fabricantes e também pingente associado a ponta cone vazio. As velocidades de deslocamento do pulverizador avaliadas foram 20 e $27 \mathrm{~km} \mathrm{~h}^{-1}$. As taxas de aplicações foram de 50 e $80 \mathrm{~L} \mathrm{ha}^{-1}$. Os parâmetros avaliados foram: DMV, amplitude relativa (dispersão), volume, densidade e cobertura. O DMV foi satisfatório para todos os tratamentos. A ponta P2 obteve resultados inferiores nos parâmetros densidade de gotas, DMV e dispersão. Na velocidade de $27 \mathrm{~km} \mathrm{~h}^{-1}$ os parâmetros volume de aplicação, densidade e cobertura obtiveram melhores resultados em comparação com a velocidade de $20 \mathrm{~km} \mathrm{~h}^{-1}$. A taxa de aplicação mais elevada melhorou os parâmetros de qualidade de aplicação na densidade de gotas, cobertura e volume. O acessório pingente não obteve resultado significativo para densidade, dispersão, volume e cobertura na cultura do algodoeiro.

Palavras-chave: algodão, qualidade de gotas, tecnologia de aplicação.

\section{Cone type nozzles with variation of spray rate and speed in cotton culture}

\begin{abstract}
Ramularia is one of the main fungal diseases of cotton, with serious consequences for its productivity and fiber quality. In order to reduce costs and improve the parameters of the application, the objective of this work is to test different types of conical and pendant jet tips, varying the rate of application and speed of cotton spraying. We used empty cone tips filled with different manufacturers and also pendant associated with empty cone tip The velocities evaluated were 20 and $27 \mathrm{~km} \mathrm{~h}^{-1}$. The application rates were 50 and $80 \mathrm{~L} \mathrm{ha}^{-}$ 1. The parameters evaluated were: DMV, relative amplitude (dispersion), volume, density and coverage. DMV was satisfactory for all treatments. The full cone jet tip obtained lower results in the parameters of droplet density, DMV and dispersion. At the top speed the parameters application volume, density and coverage obtained better results compared to the lowest speed. The higher application rate improved the application quality parameters in droplet density, coverage and volume. The pendant accessory did not obtain significant results for density, dispersion, volume and coverage in the cotton crop.
\end{abstract}

Keywords: cotton, drops quality, application technology.

\section{INTRODUÇÃO}

A região Centro-Oeste, principal produtora nacional de algodão com a área estimada de plantio de 841,2 mil hectares, sendo o estado de Mato Grosso o maior produtor nacional com 3225,5 milhões de toneladas (CONAB, 2017).

A cultura do algodoeiro (Gossypium hirsutum L.) apresenta inúmeros problemas em relação ao controle fitossanitário. É importante que se adote práticas eficientes que garantem o sucesso da produção possibilitando seu aumento e sustentabilidade do tratamento fitossanitário (CAVALIERI, 2013).

Devido ao Mato Grosso possuir clima e relevo favoráveis a esta cultura, o estado vem se destacando em produtividade, mecanização e tecnologia de ponta (CONAB, 2017). Visto isso, é essencial que a tecnologia de aplicação seja melhorada para evitar gastos excessivos com defensivos agrícolas, aumentando sua produção e qualidade da fibra.
Uma das principais doenças do algodoeiro é a ramulária causada pelo fungo Ramularia areola Atk. Por ter um clima favorável, no Cerrado brasileiro é comum o desenvolvimento deste patógeno. O que justifica o uso da pulverização intensa para o controle químico evitando maiores perdas ou baixa qualidade na fibra do algodão (CHITARRA, 2008).

Segundo Antuniassi; Boller (2011); (CUNHA et al., 2008) o uso racional de defensivos agrícolas têm minimizado impactos ambientais, além de garantir alimentos mais saudáveis. Porém, o conhecimento do controle químico ao alvo biológico não é suficiente para garantir a eficiência da aplicação, uma vez que os mesmos dependem que a aplicação alcance este alvo, em quantidade suficiente, de forma econômica, e sem contaminação de outras áreas.

Neste sentido, os produtores dão relevância ao produto fitossanitário a ser aplicado e pouca importância à tecnologia de aplicação. 
Os bicos geradores das gotas da pulverização são o principal componente de um pulverizador, devido que é deles que depende a vazão da barra, a taxa de aplicação, tamanho de gotas geradas (ANTUNIASSI; BOLLER, 2011).

A taxa de aplicação é a quantidade de volume de calda que é aplicado por hectare e esta influencia na deposição das gotas, não possui um valor pré-estabelecido devido ser dependente de outros fatores como: tipo de ponta empregada, condições do clima, produto a ser pulverizado, entre outros. O volume de aplicação é consequência da aplicação e não o objetivo, ou seja, o importante é a cobertura desejada no alvo pretendido (ANDEF, 2004).

Segundo Viana et al. (2010) a amplitude relativa (AR), o diâmetro médio volumétrico (DMV) e a porcentagem de gotas com diâmetros menores que $100 \mu \mathrm{m}$ são os principais parâmetros para determinação da população de gotas, bem como a qualidade da aplicação. Estes parâmetros determinam o potencial de deriva, tamanho e homogeneidade das gotas produzidas pela ponta utilizada na pulverização. Os valores de DMV e amplitude relativa necessitam ser avaliados conjuntamente para caracterizar a pulverização.

A amplitude relativa (AR) é determinada pela Equação 1, tal que: $\mathrm{Dv}_{0,1}$ - diâmetro de gota onde $10 \%$ do volume pulverizado é composto por gotas de tamanho inferior a esse valor, $\mathrm{Dv}_{0,5}$ - diâmetro de gota onde $50 \%$ do volume é constituído por gotas inferiores a esse valor (DMV), Dv $0,9-$ diâmetro de gota onde $90 \%$ do volume é composto por gotas inferiores a esse valor.

$$
A R=\frac{\mathrm{Dv} 0,9-\mathrm{Dv} 0,1}{\mathrm{Dv}, 5}
$$

(Equação 1)

Para uma correta seleção de pontas é importante avaliar o tamanho de gotas produzidas pela pulverização, de forma que esta, interfere na dinâmica da névoa produzida, na deriva, na evaporação, na capacidade de penetração no interior do dossel das culturas e de deposição sobre os alvos dos tratamentos fitossanitários. Para classificação de uma ponta é avaliado o diâmetro mediano volumétrico (DMV) das gotas produzidas medido em micrometros $(\mu \mathrm{m})$, podendo ser "muito finas", "finas", "médias", "grossas" e "muito grossas". Conceitualmente o DMV é o diâmetro que divide o volume pulverizado em duas partes iguais e, assim, poucas gotas diâmetros maiores totalizam o mesmo volume que muitas gotas de diâmetros inferiores (ANTUNIASSI; BOLLER, 2011).

A taxa de aplicação comum no passado recente era a utilização de taxas superiores a $200 \mathrm{~L} \mathrm{ha}^{-1}$. Porém, existe uma tendência a se reduzir esta, o que diminui os custos de aplicação e aumenta a eficiência (CHECHETTO et al., 2014)

Muitas dúvidas relativas a qualidade de aplicação ligadas a elevação da velocidade de deslocamento do equipamento, taxa de aplicação, velocidade e sentido do vento ainda não estão bem esclarecidas, devido a uma grande quantidade de variáveis implícita nessas variáveis (MACHADO, 2014).

O objetivo do trabalho foi avaliar diferentes tipos de pontas de jato cônico e pingente em condições de campo, variando a taxa de aplicação e velocidade a fim de encontrar melhores indicadores de qualidade na pulverização do algodoeiro.

\section{MATERIAL E MÉTODOS}

O trabalho foi realizado na fazenda Ribeiro do Céu pertencente ao Grupo Terra Santa Agro S/A localizada no município de Nova Mutum - MT nas coordenadas médias $13^{\circ} 95^{\prime} \mathrm{S}$ e $55^{\circ} 78^{\prime} \mathrm{W}$, com temperatura de $27,8^{\circ} \mathrm{C}$, velocidade do vento de $5,5 \mathrm{Km} \mathrm{h}^{-1}$ e umidade relativa de $30 \%$, dados da estação meteorológica da fazenda. Utilizando um pulverizador autopropelido da marca John Deere modelo 4730 com $245 \mathrm{Hp}$ e capacidade do reservatório para 3000 litros, com uma barra de pulverização de trinta metros composta por 60 bicos de espaçamento $0,5 \mathrm{~m}$.

A cultivar utilizada no experimento foi a 967 da Bayer, com espaçamento de 0,76 metros, estando em uma altura média de $1,30 \mathrm{~m}$. A pulverização foi realizada com o fungicida de contato Mertin da marca Syngenta na dose $0,5 \mathrm{~L} \mathrm{ha}^{-1}$ para o combate da ramulária.

A tabela 1 apresenta as pontas utilizadas e a figura 3 mostra o formato do pingente Lechler associado com a ponta cone vazio da marca Hypro 30HCX10.

Tabela 1. Pontas utilizadas e tipo de jato cônico. Table 1. Used tips and conical jet type.

\begin{tabular}{ccc}
\hline Pontas & Tipo de jato & Marcas \\
\hline P1 & Cone vazio & TeeJet Tx \\
P2 & Cone cheio & MagnoJet CH100 \\
P3 & Cone vazio & Hypro 30HCX10 \\
P4 & Cone vazio & Pingente Lechler e ponta \\
& & Hypro 30HCX10 \\
\hline
\end{tabular}

A Figura 1 mostra a disposição do acessório pingente na barra de pulverização, lançando jato de baixo para cima. As velocidades de avanço do pulverizador avaliadas foram: V1 $20 \mathrm{~km} \mathrm{~h}^{-1}$ e V2 - $27 \mathrm{~km} \mathrm{~h}^{-1}$. Os volumes de aplicações foram de T1 - $50 \mathrm{~L} \mathrm{ha}^{-1}$ e T2 - $80 \mathrm{~L} \mathrm{ha}^{-1}$. E a altura da barra usada em relação à altura da cultura do algodão foi de 0,5 metros.

Inicialmente as pontas foram submetidas as pressões de pulverização de 3 bar para conferir informações obtidas pelos fabricantes sobre os intervalos de vazão de cada uma, garantindo que seus limites não ultrapassariam o volume de aplicação almejado. Utilizando câmaras de borrachas para o direcionamento da pulverização para o acoplamento nos bicos foi conseguido todo volume de calda expelido pelas pontas em baldes de plástico resistente, coletando essa calda no tempo de um minuto seguindo a metodologia de Gandolfo et al. (2013).

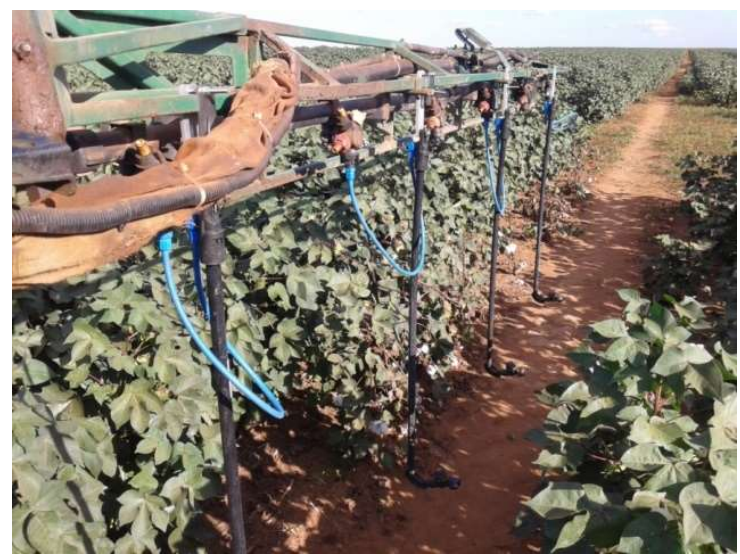

Figura 1. Disposição do Pingente Lechler associado com a ponta Hypro 30HCX10.

Figure 1. Arrangement of the Lechler Pendant associated with the Hypro 30HCX10 tip.

Para determinar o espectro das gotas de pulverização, foram usados papéis hidrossensíveis da marca Syngenta. Os papéis foram dispostos nas linhas de plantio no dossel das 
plantas (terços superior, médio e inferior) utilizando fita duplaface com o auxílio de pregadores para fixação dos papéis nas folhas do algodoeiro para melhor aproximação da realidade da aplicação.

Segundo Baesso et al. (2014) deve-se alocar as etiquetas nas posições em que se deseja analisar o espectro de gotas da pulverização. Após o procedimento, as etiquetas necessitam ser identificadas e fotografadas ou escaneadas para em seguida, fazer seu processamento.

Após a pulverização os papéis foram colhidos e dispostos em um caderno com a devida identificação de cada tratamento, escaneados por uma impressora da marca Hp multifuncional 600x600 dpi ImageRET 3600 para posterior análise pelo programa Gotas Embrapa (2000) para análise de deposição de agrotóxicos.

O delineamento adotado foi em faixas no esquema de parcelas subdivididas com 4 repetições e 16 fatores, totalizando 64 parcelas. Os dados foram submetidos a análise de variância pelo teste de Scoot-Knott aplicado a 5\% de probabilidade para a comparação de médias. Para a determinação estatística fez-se o uso do programa Sisvar, versão 5.6.

\section{RESULTADOS}

Após analisadas as amostras e feito estatísticas, obteve os resultados da Tabela 2 a seguir.

Tabela 2. Análise de variância, aplicado a $5 \%$ de probabilidade para comparação de médias pelo teste de Scoot-Knott.

Table 2. Analysis of variance, applied to $5 \%$ probability for comparison of means by the Scoot-Knott test.

\begin{tabular}{|c|c|c|c|c|c|}
\hline$P$ & DIS & VOL & DEN & COB & DMV \\
\hline P1 & $0,66 \mathrm{~b}$ & $11,72 \mathrm{a}$ & $46,18 \mathrm{a}$ & $3,44 \mathrm{a}$ & $192,03 \mathrm{~b}$ \\
\hline $\mathrm{P} 2$ & $0,72 \mathrm{a}$ & $12,93 \mathrm{a}$ & $32,02 \mathrm{~b}$ & $3,30 \mathrm{a}$ & $246,49 \mathrm{a}$ \\
\hline P3 & $0,60 \mathrm{~b}$ & $11,77 \mathrm{a}$ & $51,47 \mathrm{a}$ & $3,55 \mathrm{a}$ & $196,82 \mathrm{~b}$ \\
\hline $\mathrm{P} 4$ & $0,62 \mathrm{~b}$ & $10,71 \mathrm{a}$ & $46,56 \mathrm{a}$ & $3,22 \mathrm{a}$ & $199,83 \mathrm{~b}$ \\
\hline \multicolumn{6}{|l|}{ Vel } \\
\hline V1 & $0,65 \mathrm{a}$ & $8,02 \mathrm{~b}$ & $30,21 \mathrm{~b}$ & $2,30 \mathrm{~b}$ & $211,80 \mathrm{a}$ \\
\hline $\mathrm{V} 2$ & $0,65 \mathrm{a}$ & $15,54 \mathrm{a}$ & $57,91 \mathrm{a}$ & $4,46 \mathrm{a}$ & $205,78 \mathrm{a}$ \\
\hline \multicolumn{6}{|l|}{$\mathrm{Ta}$} \\
\hline T1 & $0,58 \mathrm{~b}$ & $7,23 \mathrm{~b}$ & $25,17 \mathrm{~b}$ & $2,04 \mathrm{~b}$ & $213,43 \mathrm{a}$ \\
\hline $\mathrm{T} 2$ & $0,73 \mathrm{a}$ & $16,33 \mathrm{a}$ & $62,95 \mathrm{a}$ & $4,72 \mathrm{a}$ & $205,78 \mathrm{a}$ \\
\hline \multicolumn{6}{|l|}{$\mathrm{A}$} \\
\hline Sup & $0,70 \mathrm{a}$ & $16,83 \mathrm{a}$ & $61,83 \mathrm{a}$ & $4,80 \mathrm{a}$ & $215,45 \mathrm{a}$ \\
\hline Méd & $0,65 \mathrm{~b}$ & $11,87 \mathrm{~b}$ & $45,74 \mathrm{~b}$ & $3,44 \mathrm{~b}$ & $205.40 \mathrm{a}$ \\
\hline Inf & $0,61 \mathrm{~b}$ & $6,64 \mathrm{c}$ & $24,60 \mathrm{c}$ & $1,90 \mathrm{c}$ & $205,52 \mathrm{a}$ \\
\hline \multicolumn{6}{|l|}{ Teste F } \\
\hline$P$ & $3,10^{*}$ & $0,72^{\mathrm{ns}}$ & $3,15 *$ & $0,21^{\mathrm{ns}}$ & $27.63^{*}$ \\
\hline V & $0,00^{\mathrm{ns}}$ & $50,04 *$ & $34,49 *$ & $48,1 *$ & $1,55^{\mathrm{ns}}$ \\
\hline $\mathrm{Ta}$ & $25,8^{*}$ & $73,03 *$ & $64,17 *$ & $73,7^{*}$ & $3,71^{\mathrm{ns}}$ \\
\hline A & $3,74^{*}$ & $20,61 *$ & $25,20 *$ & $22,2 *$ & $1,25^{\mathrm{ns}}$ \\
\hline $\mathrm{PxV}$ & $0,52^{\mathrm{ns}}$ & $4,48^{*}$ & $1,79^{\mathrm{ns}}$ & $3,67 *$ & $3,94 *$ \\
\hline PxTa & $0,24^{\mathrm{ns}}$ & $1,89^{\mathrm{ns}}$ & $3,37 *$ & $2,43^{\mathrm{ns}}$ & $0,51^{\mathrm{ns}}$ \\
\hline VxTa & $3,98^{\text {ns }}$ & $1,40^{\mathrm{ns}}$ & $4,42 *$ & $1,95^{\mathrm{ns}}$ & $2,46^{*}$ \\
\hline PxVxTa & $1,65^{\mathrm{ns}}$ & $3,33^{*}$ & $1,22^{\mathrm{ns}}$ & $2,80^{\mathrm{ns}}$ & $0,71^{\mathrm{ns}}$ \\
\hline $\mathrm{AxP}$ & $1,26^{\mathrm{ns}}$ & $0,2^{\mathrm{ns}}$ & $0,39^{\mathrm{ns}}$ & $0,68^{\mathrm{ns}}$ & $1,08^{\mathrm{ns}}$ \\
\hline $\mathrm{AxV}$ & $0,48^{\mathrm{ns}}$ & $2,02^{\mathrm{ns}}$ & $2,26^{\mathrm{ns}}$ & $2,12^{\mathrm{ns}}$ & $0,47^{\mathrm{ns}}$ \\
\hline AxTa & $1,61^{\mathrm{ns}}$ & $5,05^{*}$ & $6,64 *$ & $5,63^{*}$ & $1,42^{\text {ns }}$ \\
\hline $\mathrm{AxPxV}$ & $0,6^{\mathrm{ns}}$ & $0,25^{\mathrm{ns}}$ & $1,11^{\mathrm{ns}}$ & $0,35^{\mathrm{ns}}$ & $0,48^{\mathrm{ns}}$ \\
\hline AxPxTa & $1,27^{\mathrm{ns}}$ & $1,07^{\mathrm{ns}}$ & $2,03 *$ & $1,34^{\mathrm{ns}}$ & $0,86^{\mathrm{ns}}$ \\
\hline $\mathrm{AxVxTa}$ & $2,35^{\mathrm{ns}}$ & $0,11^{\mathrm{ns}}$ & $0,70^{\mathrm{ns}}$ & $0,10^{\mathrm{ns}}$ & $3,17 *$ \\
\hline $\mathrm{AxPxVxTa}$ & $1,27^{\mathrm{ns}}$ & $2,87 *$ & $2,95 *$ & $2,77 *$ & $0,48^{\text {ns }}$ \\
\hline $\mathrm{CV} \%$ & 27,28 & 54,17 & 64,22 & 55,26 & 13,85 \\
\hline
\end{tabular}

P: Pontas utilizadas; DIS: dispersão ou amplitude relativa (AR); VOL: volume (L ha ${ }^{-1}$ ); DEN: densidade (gotas $\mathrm{cm}^{-2}$ ); COB: cobertura (\%); DMV: diâmetro médio volumétrico $(\mu \mathrm{m})$; Vel: velocidade de aplicação; Ta: taxa de aplicação, A: altura dos papéis hidrosenssíveis nos terços superior, médio e inferior do algodoeiro. Pelo teste de Scott-Knott à 5\% de probabilidade de erro, as médias seguidas de mesma letra não diferem entre si. Ns: não significativo *Significativo à $5 \%$ de probabilidade de erro. CV: Coeficiente de variação.

A Tabela 3 apresenta de acordo com ANDEF (2004) a classificação das gotas da pulverização de acordo com os tamanhos, e cada intervalo possui uma melhor recomendação na aplicação de defensivos.

Analisando os resultados do DMV (Tabela 2) de cada ponta os resultados estão dentro do recomendado pela ANDEF (2004) para fungicida, com classificação de tamanho de gotas média.

A Tabela 4 mostra a análise de desdobramento das alturas dentro de cada nível da interação ponta, velocidade e taxa de aplicação para o volume aplicado. Podendo ser observado que tratamentos a maior taxa de aplicação de $80 \mathrm{~L} \mathrm{ha}^{-1}$ (T2) obtiveram os maiores valores volumétricos.

A Tabela 5 apresenta a variável densidade $\left(\right.$ gotas $\left.\mathrm{cm}^{-2}\right)$ e 0 desdobramento de altura dentro de cada nível de ponta, velocidade e taxa de aplicação. A densidade está relacionada com o volume aplicado e diretamente com o bico usado na pulverização e sua competência em transformar a calda em um número de gotas maior para alcançar o alvo desejado de forma homogênea.

A Tabela 6 apresenta as recomendações de densidade de gotas para agrotóxicos segundo a ANDEF (2004). E comparando com os resultados de densidade da Tabela 5 o tratamento P1V1T1, P2V1T1, P3V1T1 e P4V1T1 ficaram muito abaixo do recomendado, não correspondendo a densidade mínima indicada para fungicida que pode variar de 50 a 70 gotas $\mathrm{cm}^{-2}$.

Tabela 3. Classificação de gotas de pulverização segundo ANDEF (2004)

Table 3. Classification of spray droplets according to ANDEF (2004)

\begin{tabular}{ccc}
\hline Classe de Pulverização & DMV $(\mu \mathrm{m})$ & Recomendação \\
\hline Muito fina & $<100$ & Fungicidas, \\
Fina & $100-175$ & Inseticidas e \\
Média & $175-250$ & $\begin{array}{c}\text { Herbicidas de } \\
\text { contato. }\end{array}$ \\
\hline Grossa & $250-375$ & Dessecação \\
Muito Grossa & $375-450$ & com herbicidas \\
Extremamente grossa & $>450$ & sistêmicos. \\
\hline
\end{tabular}

Tabela 4. Desdobramento da interação altura dentro de cada nível de ponta, velocidade e taxa de aplicação em relação ao volume. Table 4. Depopulation of the interaction height within each tip level, velocity and rate of application in relation to volume.

\begin{tabular}{cccc}
\multicolumn{4}{c}{ Volume $\left(\mathrm{L} \mathrm{ha}^{-1}\right)$} \\
\hline Tratamentos & Superior & Médio & Inferior \\
P1V1T1 & $3,75 \mathrm{~A}$ & $1,15 \mathrm{~A}$ & $1,56 \mathrm{~A}$ \\
P1V1T2 & $11,65 \mathrm{~A}$ & $10,06 \mathrm{~A}$ & $2,23 \mathrm{~A}$ \\
P1V2T1 & $14,29 \mathrm{~A}$ & $7,40 \mathrm{~A}$ & $12,05 \mathrm{~A}$ \\
P1V2T2 & $36,41 \mathrm{~A}$ & $30,60 \mathrm{~A}$ & $9,54 \mathrm{~B}$ \\
P2 V1T1 & $4,60 \mathrm{~A}$ & $3,50 \mathrm{~A}$ & $3,13 \mathrm{~A}$ \\
P2 V1T2 & $24,38 \mathrm{~A}$ & $12,39 \mathrm{~B}$ & $3,64 \mathrm{~B}$ \\
P2 V2T1 & $31,10 \mathrm{~A}$ & $13,99 \mathrm{~B}$ & $6,91 \mathrm{~B}$ \\
P2 V2T2 & $17,70 \mathrm{~A}$ & $20,86 \mathrm{~A}$ & $12,91 \mathrm{~A}$ \\
P3 V1T1 & $2,56 \mathrm{~A}$ & $2,32 \mathrm{~A}$ & $2,48 \mathrm{~A}$ \\
P3 V1T2 & $23,26 \mathrm{~A}$ & $18,01 \mathrm{~A}$ & $6,44 \mathrm{~B}$ \\
P3 V2T1 & $11,74 \mathrm{~A}$ & $10,54 \mathrm{~A}$ & $7,19 \mathrm{~A}$ \\
P3 V2T2 & $29,26 \mathrm{~A}$ & $17,30 \mathrm{~B}$ & $10,11 \mathrm{~B}$ \\
P4 V1T1 & $5,65 \mathrm{~A}$ & $1,23 \mathrm{~A}$ & $2,13 \mathrm{~A}$ \\
P4 V1T2 & $14,44 \mathrm{~A}$ & $20,03 \mathrm{~A}$ & $11,81 \mathrm{~A}$ \\
P4 V2T1 & $10,55 \mathrm{~A}$ & $9,34 \mathrm{~A}$ & $4,44 \mathrm{~A}$ \\
P4 V2T2 & $28,00 \mathrm{~A}$ & $11,10 \mathrm{~B}$ & $9,74 \mathrm{~B}$ \\
\hline
\end{tabular}

Médias seguidas de mesma letra maiúsculas nas colunas, não diferem entre si pelo teste de Scott-Knott, à $5 \%$ de probabilidade de erro. 
Tabela 5. Desdobramento da interação altura dentro de cada nível de ponta, velocidade e taxa de aplicação em relação a densidade.

Table 5. Depopulation of the interaction height within each tip level, speed and rate of application in relation to density.

\begin{tabular}{cccc}
\hline \multicolumn{4}{c}{ Densidade $\left(\right.$ gotas $\left.^{-2}\right)$} \\
\hline Tratamentos & Superior & Médio & Inferior \\
P1V1T1 & $10,59 \mathrm{~A}$ & $4,13 \mathrm{~A}$ & $6,17 \mathrm{~A}$ \\
P1V1T2 & $59,09 \mathrm{~A}$ & $46,55 \mathrm{~A}$ & $18,55 \mathrm{~A}$ \\
P1V2T1 & $61,75 \mathrm{~A}$ & $32,74 \mathrm{~A}$ & $42,75 \mathrm{~A}$ \\
P1V2T2 & $122,30 \mathrm{~A}$ & $116,00 \mathrm{~A}$ & $33,58 \mathrm{~B}$ \\
P2 V1T1 & $7,58 \mathrm{~A}$ & $6,35 \mathrm{~A}$ & $3,94 \mathrm{~A}$ \\
P2 V1T2 & $62,14 \mathrm{~A}$ & $35,12 \mathrm{~A}$ & $13,47 \mathrm{~A}$ \\
P2 V2T1 & $84,71 \mathrm{~A}$ & $32,51 \mathrm{~B}$ & $18,64 \mathrm{~B}$ \\
P2 V2T2 & $58,90 \mathrm{~A}$ & $35,00 \mathrm{~A}$ & $25,90 \mathrm{~A}$ \\
P3 V1T1 & $4,61 \mathrm{~A}$ & $4,11 \mathrm{~A}$ & $5,60 \mathrm{~A}$ \\
P3 V1T2 & $122,89 \mathrm{~A}$ & $56,45 \mathrm{~B}$ & $23,12 \mathrm{~B}$ \\
P3 V2T1 & $60,73 \mathrm{~A}$ & $40,08 \mathrm{~A}$ & $40,43 \mathrm{~A}$ \\
P3 V2T2 & $118,26 \mathrm{~A}$ & $98,01 \mathrm{~B}$ & $43,39 \mathrm{~B}$ \\
P4 V1T1 & $15,23 \mathrm{~A}$ & $4,63 \mathrm{~A}$ & $3,35 \mathrm{~A}$ \\
P4 V1T2 & $55,58 \mathrm{~B}$ & $100,78 \mathrm{~A}$ & $54,98 \mathrm{~B}$ \\
P4 V2T1 & $56,08 \mathrm{~A}$ & $44,01 \mathrm{~A}$ & $13,32 \mathrm{~A}$ \\
P4 V2T2 & $112,81 \mathrm{~A}$ & $51,48 \mathrm{~B}$ & $46,45 \mathrm{~B}$ \\
\hline
\end{tabular}

Médias seguidas de mesma letra maiúscula nas colunas, não diferem entre $\mathrm{s}$ pelo teste de Scott-Knott, à $5 \%$ de probabilidade de erro.

Tabela 6. Parâmetros de densidade de gotas aconselháveis para agrotóxicos (ANDEF, 2004).

Table 6. Recommended Density Parameters for Agrochemicals (ANDEF, 2004)

\begin{tabular}{cc}
\hline Densidade $\left(\right.$ gotas $\left.\mathrm{cm}^{-2}\right)$ & Pulverização \\
\hline $20-30$ & Média - Grossa \\
$50-70$ & Média - Fina \\
$70-100$ & Fina \\
\hline
\end{tabular}

A Tabela 7 exibe a porcentagem de cobertura e 0 desdobramento de altura dentro de casa nível de ponta, velocidade e taxa de aplicação.

Tabela 7. Desdobramento da interação altura dentro de cada nível de ponta, velocidade e taxa de aplicação em relação a cobertura.

Table 7. Depopulation of the interaction height within each tip level, speed and application rate in relation to the coverage.

\begin{tabular}{cccc}
\hline \multicolumn{4}{c}{ Cobertura $(\%)$} \\
\hline Tratamentos & Superior & Médio & Inferior \\
P1V1T1 & $0,47 \mathrm{~A}$ & $0,34 \mathrm{~A}$ & $1,06 \mathrm{~A}$ \\
P1V1T2 & $3,61 \mathrm{~A}$ & $3,07 \mathrm{~A}$ & $0,74 \mathrm{~A}$ \\
P1V2T1 & $4,32 \mathrm{~A}$ & $2,19 \mathrm{~A}$ & $3,51 \mathrm{~A}$ \\
P1V2T2 & $10,30 \mathrm{~A}$ & $8,94 \mathrm{~A}$ & $2,71 \mathrm{~B}$ \\
P2 V1T1 & $1,13 \mathrm{~A}$ & $0,85 \mathrm{~A}$ & $0,72 \mathrm{~A}$ \\
P2 V1T2 & $6,19 \mathrm{~A}$ & $3,30 \mathrm{~B}$ & $1,01 \mathrm{~B}$ \\
P2 V2T1 & $3,68 \mathrm{~A}$ & $3,55 \mathrm{~B}$ & $1,72 \mathrm{~B}$ \\
P2 V2T2 & $4,38 \mathrm{~A}$ & $5,47 \mathrm{~A}$ & $3,17 \mathrm{~A}$ \\
P3 V1T1 & $0,64 \mathrm{~A}$ & $0,57 \mathrm{~A}$ & $0,66 \mathrm{~A}$ \\
P3 V1T2 & $7,35 \mathrm{~A}$ & $5,06 \mathrm{~A}$ & $1,89 \mathrm{~B}$ \\
P3 V2T1 & $3,74 \mathrm{~A}$ & $3,13 \mathrm{~A}$ & $2,40 \mathrm{~A}$ \\
P3 V2T2 & $8,53 \mathrm{~A}$ & $5,66 \mathrm{~B}$ & $2,99 \mathrm{~B}$ \\
P4 V1T1 & $1,59 \mathrm{~A}$ & $0,38 \mathrm{~A}$ & $0,51 \mathrm{~A}$ \\
P4 V1T2 & $4,22 \mathrm{~A}$ & $6,18 \mathrm{~A}$ & $3,61 \mathrm{~A}$ \\
P4 V2T1 & $3,32 \mathrm{~A}$ & $2,89 \mathrm{~A}$ & $1,23 \mathrm{~A}$ \\
P4 V2T2 & $8,31 \mathrm{~A}$ & $3,42 \mathrm{~B}$ & $3,05 \mathrm{~B}$ \\
\hline
\end{tabular}

Médias seguidas de mesma letra maiúscula nas colunas, não diferem entre si pelo teste de Scott-Knott, à $5 \%$ de probabilidade de erro.

\section{DISCUSSÃO}

Podemos verificar pelos resultados que as pontas não apresentaram resultados significativos nos parâmetros volume e cobertura, a velocidade não apresentou significância para os parâmetros dispersão e DMV (Tabela 2). A taxa de aplicação e as alturas não apresentaram significativos resultados para DMV.
Para comparação do parâmetro de dispersão quanto menor o valor tendendo a zero mais homogêneo é considerada a aplicação. Diante disto, observa-se que a ponta (P2) obteve o pior desempenho, e pelo resultado estatístico as pontas P1, P3 e P4 não se diferenciaram.

$\mathrm{Na}$ velocidade de deslocamento V2 (Tabela 2), observa-se uma melhora na cobertura, densidade de gotas e volume referente à aplicação. Isso pode ser explicado devido à redução do tamanho de gotas, pelo aumento da pressão do sistema provocado pelo sistema automático do pulverizador para compensar o aumento da velocidade de deslocamento. Os resultados corroboram com ZAIDAN (2012) em um trabalho analisou diferentes pontas na pulverização terrestre, e verificou melhora na deposição de gotas na parte superior das plantas em velocidade de deslocamento mais elevadas na pulverização.

Em todo estado de Mato Grosso há uma forte tendência para redução da taxa de aplicação na qual representa economia de água, combustível e desgaste das máquinas de aplicação, porém para o correta eficácia requer otimização da tecnologia de aplicação para que mantenha uma qualidade e a eficiência das aplicações (SOUZA et al., 2012) o que torna a seleção das pontas de pulverização bastante criteriosa e importante. E geralmente os usuários não tem controle e nem conhecimento adequado para utilização da ponta correta para determinadas situações.

O trabalho demostra que o aumento na taxa de aplicação de 50 para 80 litros melhorou a dispersão, volume, densidade e cobertura (Tabela 2). Dados também confirmados por Rodrigues et al. (2010). Outro trabalho discorda, segundo Oliveira et al. (2010), utilizando diferentes taxas de aplicação não observaram diferença no controle de pragas.

O parâmetro de volume se faz necessário para ter conhecimento da quantidade da aplicação chega ao alvo desejado, e devido a isto se a aplicação será suficiente para combater doenças e pragas relacionadas à cultura cultivada. $\mathrm{O}$ trabalho demostra (Tabelas 2 e 4) que a taxa de aplicação mais elevada (T2) foi superior em termos de volume que chegou até a planta, contribuindo para o melhor controle da praga.

De acordo com (SOUZA et al., 2007) a deposição de gotas é maior nas folhas mais altas e diminui para as folhas médias e inferiores, onde ocorre maior frequência de doenças fúngicas no algodoeiro, os resultados conferem com os demostrados nesta pesquisa (Tabela 4).

Pela Tabela 5 observa-se que a altura média do tratamento P4V1T2 obteve um valor de densidade maior que a altura superior justificando que a $\mathrm{P} 4$ pingente que possui um designer que pulveriza a calda de baixo para cima. Nos demais tratamentos a densidade nas alturas superiores foram maiores que os médios e inferiores.

Analisando os resultados da Tabela 7 observou-se valores de cobertura maiores nas camadas superiores, e a taxa de aplicação influenciou, sendo observado na T2 diferenças de cobertura entre as alturas.

$\mathrm{O}$ pingente (P4) teve um comportamento de arrancar algumas maçãs do algodão que não foi contabilizado e um envergamento significativo de sua estrutura quando a velocidade foi elevada para V2. Desta forma, não correspondeu à posição ideal para aplicação no experimento. Além disto, apresentou um problema operacional no fechamento da barra do pulverizador podendo danificar os pingentes principalmente os da extremidade da barra. 


\section{CONCLUSÕES}

Para o DMV todas as pontas em todos os tratamentos obtiveram resultados satisfatórios para recomendação de fungicida.

A ponta $(\mathrm{P} 2)$ obteve resultados inferiores na análise de dispersão, densidade de gotas e DMV em relação às demais pontas.

$\mathrm{Na}$ velocidade (V2) o volume de aplicação, densidade e cobertura obtiveram melhores resultados em comparação com a velocidade $\mathrm{V} 1$.

Com a taxa de aplicação (T2) melhoraram os resultados da qualidade de aplicação relacionados com o volume, densidade de gotas e cobertura.

A utilização do pingente como acessório não obteve resultado significativo para densidade das gotas, volume, dispersão e cobertura na cultura do algodoeiro.

$\mathrm{O}$ uso do pingente deve ser mais bem estudado a sua aplicação em diferentes estádios da cultura do algodão como analise de danos nas maças e também à resistência do material do pingente em condição de campo.

\section{REFERÊNCIAS}

ANDEF_ASSOCIAÇÃO NACIONAL DE DEFESA VEGETAL. Manual de Tecnologia de Aplicação de Produtos Fitossanitários. 1. ed. São Paulo: Linea Creativa, 2004. 52 p.

ANTUNIASSI, U. R.; BOLLER, W. Tecnologia de aplicação para culturas anuais. Passo Fundo: Aldeia Norte FEPAF, 2011. 279 p. v. 1.

BAESSO, M. M. Pesticide application technologies. Revista Ceres, Viçosa, v. 61, p. 780-785, 2014. DOI: http://dx.doi.org/10.1590/0034-737x201461000003

CAVALIERI, J. D. Pontas e velocidade de deslocamento na deposição de gotas da pulverização na cultura do algodão. 2013. 82f. Dissertação (Mestrado em Agronomia) - Universidade Estadual Paulista, Botucatu, 2013.

CHECHETTO, R. G.; MOTA, A. A. B.; ANTUNIASSI, U. R.; CARVAlHO, F. K.; VILELA, C. M.; SILVA, A. C. A. Caracterização da taxa de aplicação e pontas de pulverização utilizadas no Estado de Mato Grosso Magistra, Cruz das Almas, v. 26, n. 1, p. 89-97, 2014

CHITARRA, L. G. Identificação e controle das principais doenças do algodoeiro. 3. ed. Campina Grande: Embrapa Algodão. 88 p. (Cartilha, 2)

CONAB. Levantamento de safra - Setembro 2017. 2017. Disponível em: https://www.conab.gov.br/infoagro/safras/graos/boletim-da-safra-de-graos. Acesso em: 11 nov. 2018.

CUNHA, J. A. P.; MOURA, E. A. C.; SILVA JR, J. L. da; ZAGO, F. A.; JUSLIATTI, F. C. Efeito de pontas de pulverização no controle químico da ferrugem da soja. Engenharia Agrícola, Jaboticabal, v. 28, n. 2, p. 283-291, 2008. DOI: http://dx.doi.org/10.1590/S010069162008000200009

EMBRAPA_Empresa Brasileira de Pesquisa Agropecuária. Programa de Calibração de Pulverização - Gotas. $2000 . \quad$ Disponível em: $<$ https://www.embrapa.br/busca-de-produtos-processose-servicos/-/produto-servico/1421/gotas---programa-decalibracao-de-pulverizacao---gotas $>$. Acesso $16 \mathrm{de}$ Agosto de 2017.
GANDOLFO, M. A.; ANTUNIASSI, U. R.; GANDOLFO, U. D.; MORAES, E. D.; RODRIGUES, E. B.; ADEGAS, F. S. Inspeção periódica de pulverizadores: diagnóstico para a região norte do Paraná. Engenharia Agrícola, Jaboticabal, v. 33, n. 2, p. 411- 421, 2013. DOI: http://dx.doi.org/10.1590/S0100-69162013000200019

MACHADO, T. M. Inspeção periódica de pulverizadores de barras na região de Guarapuava - PR. Enciclopedia Biosfera, Goiânia, v. 10, n. 19; p. 1225-1233, 2014. DOI: http://dx.doi.org/10.14688/1984-3801/gst.v8n3p87-94

OLIVEIRA, J. R. G.; FERREIRA, M. C.; ROMÁN, R. A. A. Diferentes diâmetros de gotas e equipamentos para aplicação de Inseticida no controle de Pseudoplusia includens. Engenharia Agrícola, Jaboticabal, v. 30, n. 1, p. 92-99, 2010. DOI: http://dx.doi.org/10.1590/S010069162010000100010

RODRIGUES, A. C. P.; FILHO, S. I. B. S.; MARTINS, D.; COSTA, N. V.; ROCHA, D. C.; SOUZA, G. S. F. Avaliação qualitativa e quantitativa na deposição de calda de pulverização em Commelina benghalensis. Planta Daninha, Viçosa, v. 28, n. 2, p. 421-428, 2010. DOI: http://dx.doi.org/10.1590/S0100-83582010000200022

SOUZA, L. A.; CUNHA, J. P. A. R.; PAVANIN, L. A. Deposição do herbicida 2,4-D Amina com diferentes volumes e pontas de pulverização em plantas infestantes. Revista Ciência Agronômica, Fortaleza, v. 43, n. 1, p. 7885, 2012. DOI: http://dx.doi.org/10.1590/S180666902012000100010

SOUZA, R. T.; CASTRO, R. D.; PALLADINI, L. A. Depósito de pulverização com diferentes padrões de gotas em aplicações na cultura do algodoeiro. Engenharia Agrícola, Jaboticabal, v. 27, n. esp., p. 75-82, jan. 2007. DOI: http://dx.doi.org/10.1590/S010069162007000200011

VIANA, R. G.; FERREIRA, L. R.; FERREIRA, M. C.; TEIXEIRA, M. M.; ROSELL, J. R.; TUFFI SANTOS, L. D.; MACHADO, A. F. L. Volumetric distribution and droplet spectrum by low drift spray nozzles. Planta Daninha, Viçosa, v. 28, n. 2, p. 439-446, 2010. DOI: http://dx.doi.org/10.1590/S0100-83582010000200024

ZAIDAN, S. E. Influência nos diferentes tipos de pontas de pulverização nas aplicações terrestres em alta velocidade na cultura da soja (Glycine max). 2012. 79f. Dissertação (Mestrado em Ciências) - Escola Superior de Agricultura "Luiz de Queiroz", Piracicaba, 2012. 